\title{
Dielectric Properties of Biocompatible and Biodegradable Polycaprolone and Polylactide and Their Nanocomposites in the Millimeter Wave Band
}

\author{
V. V. Meriakri' ${ }^{1, *}$, D. S. Kalenov ${ }^{1}$, M. P. Parkhomenko ${ }^{1}$, S. Zhou ${ }^{2}$, N. A. Fedoseev ${ }^{1}$ \\ ${ }^{1}$ Kotel'nikov Institute of Radio Engineering and Electronics, Russian Academy of Sciences, Fryazino Branch, 1 Vvedenskii sq., Fryazino, \\ Moscow region, 141190 Russia \\ ${ }^{2}$ Southwest Jiaotong University, School of Materials Science and Engineering 111, 1st Section, Northern 2nd Ring Road, Chengdu, \\ Suchan, CN 610031, China
}

\begin{abstract}
Dielectric properties of biocompatible and biodegradable poly- $\varepsilon$-caprolone (PCL) and polylactide (PLA), as well as their nanocomposites, are measured in the millimeter (MM) wave band. It is found that the complex dielectric permittivity of PCL and its nanocomposites increases with temperature in the interval from 40 to $50^{\circ} \mathrm{C}$, while that of PLA and its nanocomposites increases in the interval from 85 to $90^{\circ} \mathrm{C}$. The samples of PLA with carbon nanotubes prepared with melamine show much lower absorption compared with the same samples prepared without melamine. The latter fact can be attributed to the formation of clusters of nanotubes in these samples.
\end{abstract}

Keywords Applications, Biocompatibility, Biodegradable, Dielectric Properties, Nanostructured Polymers

\section{Introduction}

Polycaprolone (PCL) and polylactide (PLA) are widely used in biomedicine for wound covering and prosthetics, including tooth implantation[1,2]. The main advantages of these materials are their biocompatibility and slow degradation accompanied by release of water and carbon dioxide; the latter fact makes these polymers a perfect ecologically pure packing material. Therefore, the study of the electrical and dielectric properties of these materials, in particular, the absorption of electromagnetic waves of different frequency bands, is of great scientific and practical interest. Data on the preparation process and material characterization, including dielectric properties of PCL and PLA, are presented elsewhere [3-16]; for example, data on the dielectric properties of pure PCL and PLA at frequencies from $10^{-1}$ to $10^{6} \mathrm{~Hz}[3,4]$, and data on the properties of PLA in the frequency band $0.5-10 \mathrm{GHz}[5]$. However, there are no data on the dielectric properties of PCL and PLA and their nanocomposites in the millimeter (MM) wave band. The importance of these data is associated, in particular, with a recent increase in the application of MM waves in medicine [29]. We have measured for the first time the dielectric properties of crosslinked poly-e-caprolone (cPCL) and PLA,

Corresponding author:

meriakri@ms.ire.rssi.ru (V. V. Meriakri)

Published online at http://journal.sapub.org/materials

Copyright (C) 2012 Scientific \& Academic Publishing. All Rights Reserved as well as their composites with carbon nanotubes (CNTs), in the frequency range from 85 to $118 \mathrm{GHz}$ and in the range of temperatures from 20 to $90^{\circ} \mathrm{C}$.

\section{Materials}

1. The samples of crosslinked poly-e-caprolone (cPCL) and its nanocomposites were fabricated at the Southwest Jiaotong University, Chengdu, PRC [26] by the reaction of PCL diol with methacrylic anhydride [27].

2. The samples of PLA and its nanocomposites were fabricated at the École Nationale Supérieure de Chimie de Lille, France. The synthesis of PLA was done by reactive extrusion via ring opening polymerization of L,L-lactide using a continuous single-stage process [28].

\section{Measurement Methods}

The propagation constant $\gamma$ of electromagnetic waves traveling in an infinite medium with complex refractive index $N=n-i \kappa$ is given by

$$
\gamma=\alpha+i \beta
$$

where the decay constant $\alpha(\mathrm{Np} / \mathrm{m})$ and the phase constant $\beta$ $\left(\mathrm{m}^{-1}\right)$ are related to the real $n$ and imaginary $\kappa$ parts of the refractive index by the formulas

$$
\begin{gathered}
\alpha=k_{0} \kappa, \\
\beta=k_{0} n .
\end{gathered}
$$


Here $k_{0}=2 \pi / \lambda$ is the wavenumber in vacuum and $\lambda$ is the wavelength in vacuum. Thus, having determined $\alpha$ and $\beta$ experimentally, we can find the parameters $n$ and $\kappa$ of the medium under test.

The measurements were carried out in the range of frequencies from 85 to $118 \mathrm{GHz}$ by a panoramic VSWR and attenuation meter based on an RG4-14 oscillator. The block-diagram of the setup is shown in Figure 1.

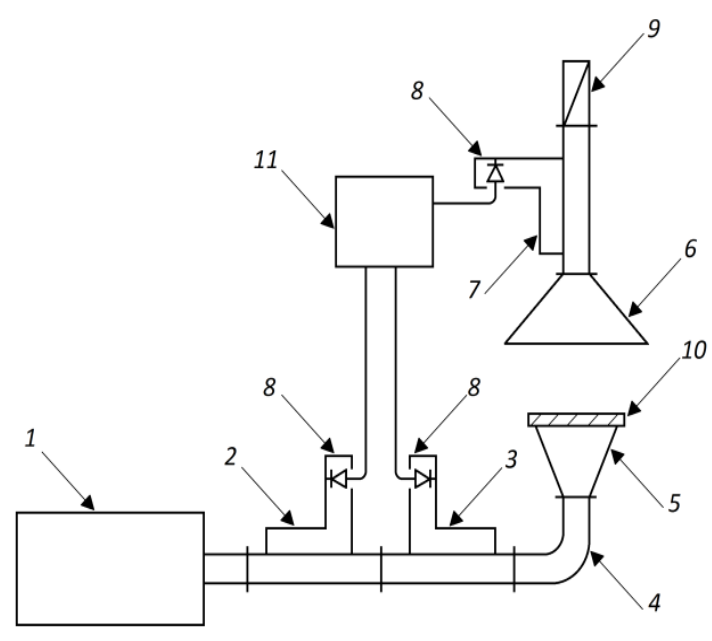

Figure 1. Block diagram of the setup

Here, 1 is a panoramic VSWR and attenuation meter based on an RG4-14 oscillator, 2 is a directional coupler for the incident wave, 3 is a directional coupler for the reflected wave, 4 is a waveguide bend, 5 is a transmitting horn, 6 is a receiving horn, 7 is a directional coupler for the transmitted wave, 8 are detector sections, 9 is a matched load, 10 is a test sample of thickness $l$, and 11 is an indicator.

The measurement setup is implemented on a rectangular waveguide of cross section of 2.4-by-1.2 $\mathrm{mm}$; the cross section of horn 5 is 10 -by-10 $\mathrm{mm}$, and the cross section of horn $6,14-\mathrm{by}-14 \mathrm{~mm}$. The increased cross section of the receiving horn allows one to receive almost all the power propagating in a spacing of $50 \mathrm{~mm}$ between the horns, where the transverse dimension of the field increases due to diffraction.

We measured the power transmission $T$ and reflection $R$ coefficients of the materials [24, 25]. For a sample thickness of $l$, which actually guarantees the absence of interference in a sample (for absorption in a sample of more than 5-7 dB), the coefficients $T$ and $R$ are related to the propagation constants by the formulas

$$
\begin{gathered}
T=(1-R) e^{-2 \alpha l}, \\
R=\left|\frac{\gamma_{0}-\gamma}{\gamma_{0}+\gamma}\right|^{2},
\end{gathered}
$$

where $\gamma_{0}=i k_{0}$ is the propagation constant in vacuum (in air).

From (4) we can determine the decay constant $\alpha$ in the medium:

$$
\alpha=\frac{\ln \left[\frac{(1-R)^{2}}{T}\right]}{2 l} .
$$

Substituting this value of $\alpha$ into (2), we obtain the imaginary part $\kappa$ of the refractive index of the sample:

$$
\kappa=\frac{\alpha}{k_{0}} \text {. }
$$

To determine the real part $n$ of the refractive index of the sample, we apply (5), which in our case is transformed to

$$
R=\frac{(1-n)^{2}+\kappa^{2}}{(1+n)^{2}+\kappa^{2}} .
$$

Solving this equation, we derive a formula for finding $n$ :

$$
n=\frac{1+R}{1-R}+\sqrt{\left(\frac{1+R}{1-R}\right)^{2}-\left(\kappa^{2}+1\right)} .
$$

The complex dielectric constant $\varepsilon=\varepsilon_{1}-i \varepsilon_{2}$ of the medium is related to its complex refractive index $N=n-i \kappa$ by the formula $N^{2}=\varepsilon$, from which we obtain an expression for determining the real $\varepsilon_{1}$ and imaginary $\varepsilon_{2}$ parts of the dielectric constant of the sample under test:

$$
\begin{gathered}
\varepsilon_{1}=n^{2}-\kappa^{2} \\
\varepsilon_{2}=2 n \kappa .
\end{gathered}
$$

\section{Measurement Results}

\subsection{Investigation of Samples of cPCL and Its Nanocomposites}

Table 1. Dielectric properties of CPLC and nanocomposites in the frequency band $90-100 \mathrm{GHz}$

\begin{tabular}{|c|c|c|c|c|c|}
\hline $\begin{array}{c}\text { Sample } \\
\text { no. }\end{array}$ & $n$ & $\kappa$ & $\varepsilon_{1}$ & $\varepsilon_{2}$ & $T,{ }^{\circ} \mathrm{C}$ \\
\hline \multirow{2}{*}{1} & 1.7 & 0.0030 & 2.9 & 0.010 & 25 \\
\cline { 2 - 6 } & 1.8 & 0.0045 & 3.2 & 0.015 & 45 \\
\hline \multirow{2}{*}{2} & 2.7 & 0.13 & 7.1 & 0.7 & 25 \\
\cline { 2 - 6 } & 2.7 & 0.16 & 7.1 & 0.8 & 45 \\
\hline \multirow{2}{*}{3} & 2.7 & 0.003 & 7.1 & 0.016 & 25 \\
\cline { 2 - 6 } & 2.7 & 0.005 & 7.1 & 0.029 & 45 \\
\hline \multirow{2}{*}{4} & 2.3 & 0.010 & 5.3 & 0.05 & 25 \\
\cline { 2 - 6 } & 2.4 & 0.017 & 5.8 & 0.08 & 45 \\
\hline
\end{tabular}

We have measured the dielectric characteristics of four samples: 1 , pure cPCL; 2 , cPCL containing $5 \%$ of multiwall CNTs (MCNTs); 3, cPCL containing $15 \%$ of $\mathrm{Fe}_{3} \mathrm{O}_{4}$; and 4, cPCL containing $3.75 \%$ of MCNTs and $11.25 \%$ of $\mathrm{Fe}_{3} \mathrm{O}_{4}$.

The dielectric properties of $\mathrm{CPCL}$ were measured in the temperature interval $T=25-45^{\circ} \mathrm{C}$. In [3], when studying pure cPCL at frequency of $100 \mathrm{kHz}$, the authors observed an increase in $\varepsilon_{2}$ with temperature in the interval from 35 to $50^{\circ} \mathrm{C}$. In our experiments carried out in the frequency band of $90100 \mathrm{GHz}$, we also observed an increase in $\varepsilon_{2}$ in this temperature interval with a maximum at $T=45^{\circ} \mathrm{C}$ in all the samples. The results of these measurements are presented in Table 1 . Note that, within the measurement accuracy $(3 \%$ for 
$n$ and $15 \%$ for $\kappa$ ), we did not observe any dielectric dispersion in this frequency band.

Table 1 shows that the maximal increase in $\kappa$ and $\varepsilon_{2}$ is caused by the introduction of carbon nanotubes into cPCL. The introduction of $\mathrm{Fe}_{3} \mathrm{O}_{4}$ did not lead to a noticeable change in the absorption of the samples.

\subsection{Investigation of Samples of PLA and Its Nanocomposites}

The results of the measurements of the dielectric properties of PLA and its nanocomposites are presented in Table 2. Here sample 1 is pure PLA, sample 2 is PLA prepared with $1 \%$ of MWNT of diameter $40-60 \mathrm{~nm}$, and length of $5-15$ $\mu \mathrm{m}$ with melamine and sample 3, PLA prepared with $1 \%$ of the same MWNT without melamine.

The measurements were carried out in the same frequency band as those of the cPCL samples but in a larger temperature interval $\left(T=25-90{ }^{\circ} \mathrm{C}\right)$, because, in the temperature interval of $50-90^{\circ} \mathrm{C}$, the authors of [3] observed an increase in the $\varepsilon_{2}$ of pure PLA with a maximum at $85^{\circ} \mathrm{C}$. We also observed an increase in $\varepsilon_{2}$ in all the samples (pure PLA and its nanocomposites) in this temperature interval.

Table 2. Dielectric properties of PLA and its nanocomposites at frequency of $\mathrm{f}=96 \mathrm{GHz}$

\begin{tabular}{|c|c|c|c|c|c|}
\hline $\begin{array}{c}\text { Sample } \\
\text { no. }\end{array}$ & $n$ & $\kappa$ & $\varepsilon_{1}$ & $\varepsilon_{2}$ & $T,{ }^{\circ} \mathrm{C}$ \\
\hline \multirow{2}{*}{1} & 1.66 & $<0.01$ & 2.76 & 0.01 & 25 \\
\cline { 2 - 6 } & 1.66 & 0.02 & 2.76 & 0.06 & 90 \\
\hline \multirow{2}{*}{2} & 4.0 & 0.40 & 15.70 & 3.20 & 26 \\
\cline { 2 - 6 } & 4.0 & 0.44 & 15.60 & 3.50 & 90 \\
\hline \multirow{2}{*}{3} & 3.7 & 0.69 & 13.40 & 5.10 & 24 \\
\cline { 2 - 6 } & 3.7 & 0.71 & 13.30 & 5.30 & 90 \\
\hline
\end{tabular}

Table 2 shows that, for the same concentration of MWNTs, the sample with melamine exhibits much lower absorption, which can be attributed to the formation of aggregates of nanotubes when preparing a sample in the absence of melamine. This assumption has been confirmed by the electron microscope examination of the samples carried out at École Nationale Supérieure de Chimie de Lille, France, by Professor S. Bourbigot.

Moreover, in the case of sample 3, we revealed a dispersion of $\varepsilon_{1}$ and $\varepsilon_{2}$ : both these functions decrease as frequency increases. For example, at frequency of $96 \mathrm{GHz}$ (see Table 2), these parameters amount to 13.4 and 5.1 at $24^{\circ} \mathrm{C}$ and 13.3 and 5.3 at $90^{\circ} \mathrm{C}$. At frequency of $116 \mathrm{GHz}, \varepsilon_{1}$ and $\varepsilon_{2}$ took the respective values of 12.4 and 4.6 at $24^{\circ} \mathrm{C}$ and 12.3 and 4.8 at $90^{\circ} \mathrm{C}$. This fact points to the Debye character of dispersion in sample 3, the Debye frequency being lower than the frequency at which the measurements are carried out.

\section{Discussion}

\subsection{Samples of cPCL and Its Nanocomposites}

Earlier we have investigated the dielectric characteristics of samples 1-4 at frequencies from $10^{-1}$ to $210^{6} \mathrm{~Hz}$ by a BDS40 broadband dielectric spectrometer (Novocontrol) [10]. The results of these measurements are presented in Figures 2-5.

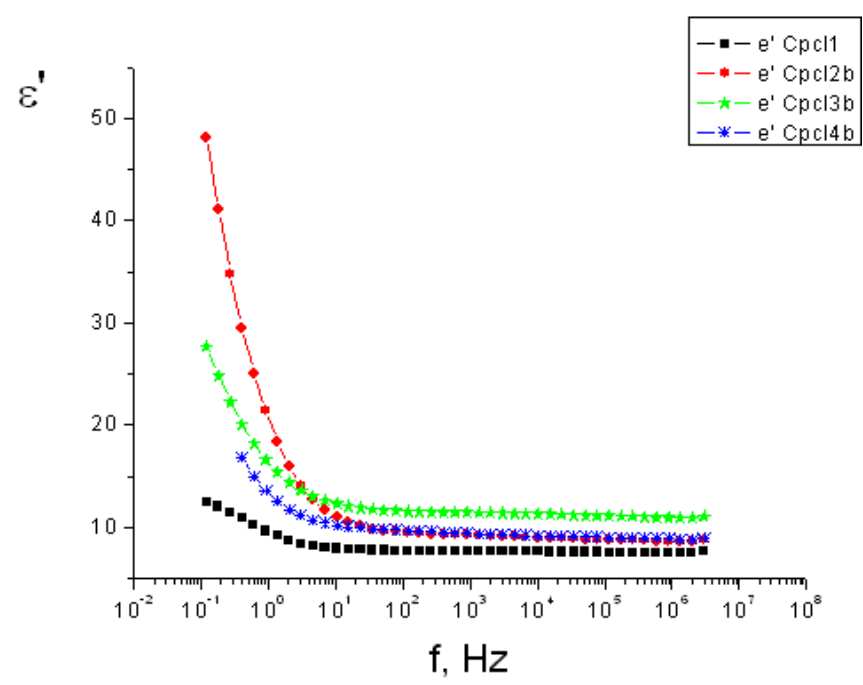

Figure 2. Real part of permittivity of cPCL and its nanocomposites at frequencies $10^{-1}-3 \cdot 10^{6} \mathrm{~Hz}$

Nanocomposites have much greater $\varepsilon_{1}$ than pure $\mathrm{CPCL}$ at frequencies of 0.1-10 Hz. For all samples, $\varepsilon_{1}$ measured at frequencies about $3.106 \mathrm{~Hz}$ is greater than the values of $\varepsilon 1$ obtained in our MM wave measurements $\left(\varepsilon_{1}=7-13\right.$ at $3 \cdot 10^{6}$ $\mathrm{Hz}$ for nanocomposites and 6 for pure $\mathrm{cPCL}$ ). This means that there is a marked dispersion of $\varepsilon_{1}$ in all samples at frequencies from $3 \cdot 10^{6} \mathrm{~Hz}$ to $80 \mathrm{GHz}$.

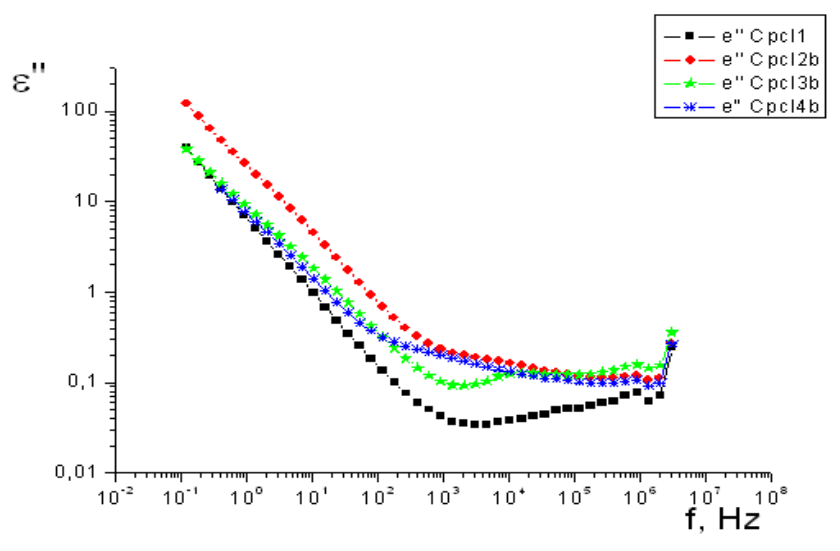

Figure 3. Imaginary part of permittivity of $\mathrm{CPCL}$ and its nanocomposites at frequencies $10^{-1}-3 \cdot 10^{6} \mathrm{~Hz}$

Figure 3 shows that, at frequencies about $10^{6} \mathrm{~Hz}$, all the samples have practically the same $\varepsilon_{2}=0.1$. Samples 1 and 3 have much less $\varepsilon_{2}$ (Table 1). On the other hand, the values of $\varepsilon_{2}$ of samples 2 and 4 , which contain multiwall CNTs, are much greater than those in Figure 3. This means that multiwall CNTs exhibit marked dispersion of $\varepsilon$ in all the samples at frequencies from $3 \cdot 10^{6} \mathrm{~Hz}$ to $80 \mathrm{GHz}$.

Figures 4 and 5 present the imaginary parts of permittivity of samples 2 and 1 at frequencies $10^{-1}-3 \cdot 10^{6} \mathrm{~Hz}$ without regard to conductivity $\sigma$. 


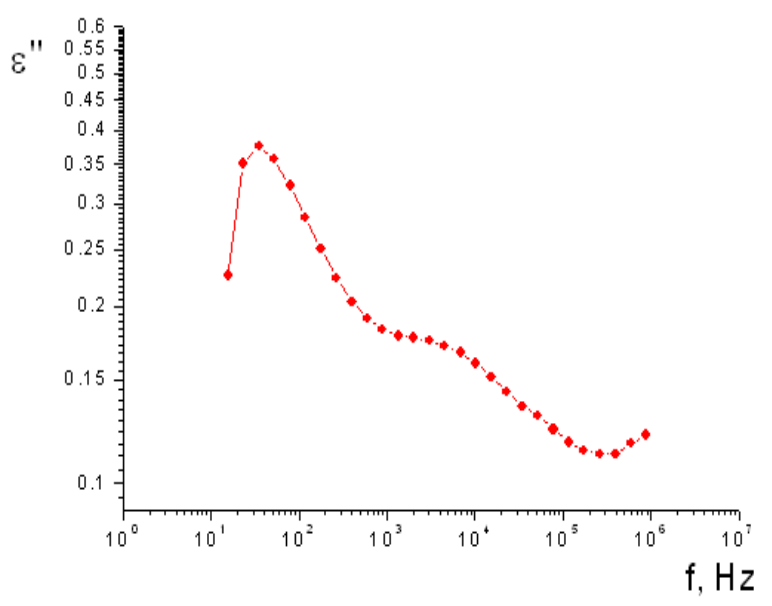

Figure 4. Imaginary part of permittivity of sample 2 at frequencies $10^{-1}-3 \cdot 10^{6} \mathrm{~Hz}$ without regard to conductivity $\sigma$

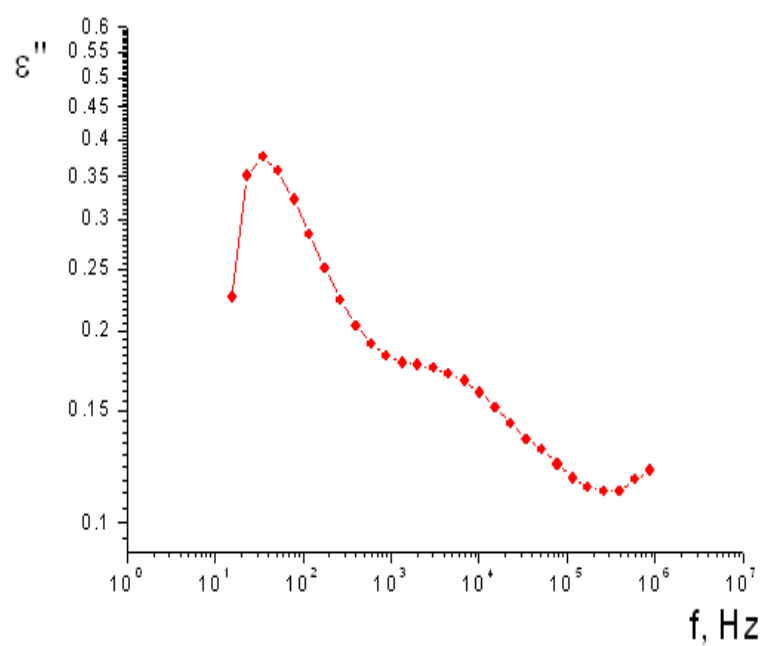

Figure 5. Imaginary part of permittivity of sample 1 at frequencies $10^{-1}-3 \cdot 10^{6} \mathrm{~Hz}$ without regard to conductivity $\sigma$

In the MM wave band (Table 1), the contribution of to $\varepsilon_{2}$ is much less. On the contrary, for sample 2 with multiwall $\mathrm{CNTs}, \varepsilon_{2}$ in the MM waves range is high due to the dielectric dispersion in multiwall CNTs at frequencies between $3 \cdot 10^{6} \mathrm{~Hz}$ and $80 \mathrm{GHz}$.

\subsection{Samples of PLA and Its Nanocomposites}

These samples were investigated by TEM microscopy at the École Nationale Supérieure de Chimie de Lille, France, by professor S. Bourbigot.

Figures 6 and 7 present $200 \mathrm{~nm}$ TEM microscope images of the samples of PLA $+1 \%$ MWNT (without melamine) and PLA $+1 \%$ MWNT (with melamine), respectively.

The sample of PLA $+1 \%$ MWNT (with melamine) is characterized by a much more uniform distribution of MWNT without aggregation of nanoparticles compared with the sample of PLA $+1 \% \mathrm{MWNT}$ (without melamine). This is the reason for the difference in $\varepsilon_{2}$ of these two samples in Table 2.

Measurements of the dielectric properties of PLA $+1 \%$ MWNT (without melamine) and PLA+1\%MWNT (with melamine) by the resonance method at frequency of 11 $\mathrm{GHz}[10]$ confirmed the difference between the absorption in these two samples.

The dielectric permittivity of PLA $+1 \% \mathrm{MWNT}$ (without melamine) at frequency of $11 \mathrm{GHz}$ is $\varepsilon_{1}=9.16$ and $\varepsilon_{2}=3.23$.

The dielectric permittivity of a PLA $+1 \% \mathrm{MWNT}$ (with melamine) at frequency of $11 \mathrm{GHz}$ is $\varepsilon_{1}=4.36$ and $\varepsilon_{2}=0.30$.

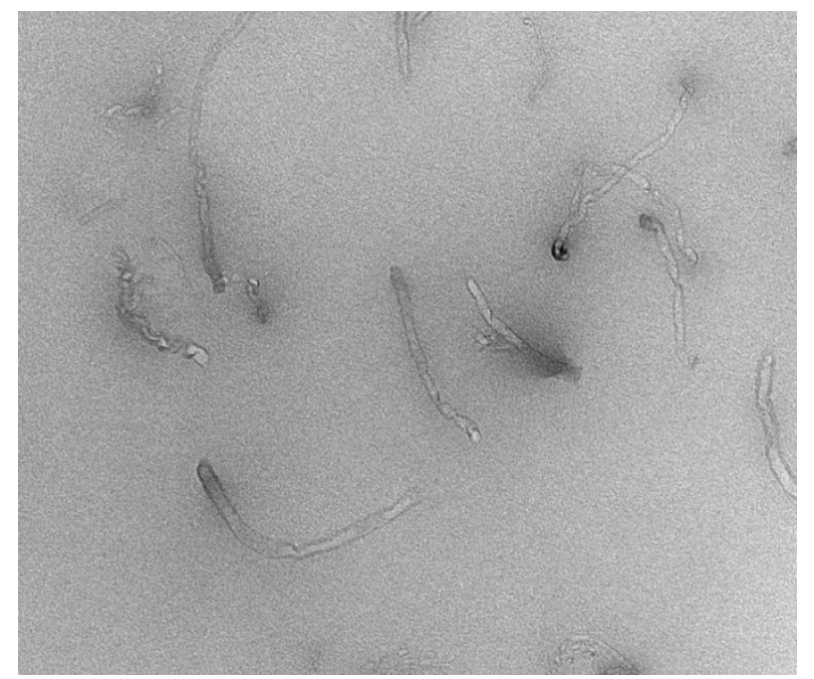

Figure 6. TEM microscopy picture of PLA $+1 \% \mathrm{MWNT}$ (without melamine) sample

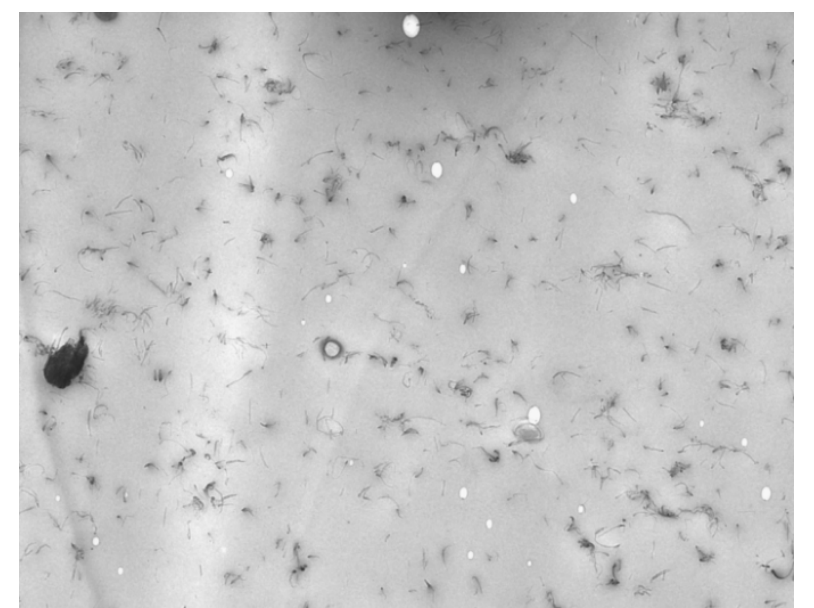

Figure 7. TEM microscopy picture of PLA $+1 \%$ MWNT (with melamine) sample

\section{Conclusions}

We have carried out the measurements of the dielectric properties of biocompatible and biodegradable crosslinked polycaprolone and polylactide polymers and their nanocomposites at frequencies from 85 to $118 \mathrm{GHz}$ and in the temperature interval in which the samples show considerable dependence of absorption of electromagnetic waves on temperature. We have found that the complex dielectric permittivity of PCL and its nanocomposites increases with temperature in the interval from 40 to $50^{\circ} \mathrm{C}$, while that of PLA and its nanocomposites increases in the interval from 85 to $90^{\circ} \mathrm{C}$. The samples of PLA with carbon 
nanotubes prepared with melamine show much lower absorption compared with the same samples prepared without melamine. The latter fact can be attributed to the formation of clusters of nanotubes in these samples.

These materials may find application, in particular, in medical diagnostics and therapy with the use of MM waves [29].

\section{ACKNOWLEDGEMENTS}

We are grateful to Professor S. Bourbigot for supplying and TEM microscopy investigation of PLA samples and useful discussions.

This work was supported by the Russian Foundation for Basic Research, project nos. 10-02-13310-RT_OMN and 11-02-93965-YuAR_a.

\section{REFERENCES}

[1] A.P. Gupta and V. Kumar: Eur. Polymer J. 43 (2007) 40530744.

[2] Y. Ikada and A. Tsuji: Macromolecular Rapid Communications, 21 (2000) 117-150.

[3] J.L.E. Ivirico, J.M.M. Dueñas, A.A. Balado, J.L.G. Ribelles and M.S. Sánchez: J. Polymer Sci. Part B: Polymer Phys. 47 (2009) 183-193.

[4] Nor'aini Ahmad Zawawi, Alyani Ismail, Abdan Khalina, and Mohd Adzir Mahdi: Key Engineering Materials 471-472 (2011) $86 \tilde{8} 873$

[5] P.Syroukal, M. Konty, V. Skelarik, P. Kucharczyk, $6^{\text {th }}$ Int. Conference on Times of Polymers (TOP) and Composites AIP Conference Proceedings 1459 (2012), Ishia, Italy, 20-22.

[6] J. Baschnagel: 2 Int. Workshop "Nonlinear Response of Soft Matter" Konstanz, Germany (2011) 70-71.

[7] A. Bur, Y. Lee, S. Roth, P. Srart: Polymer 46 (24): (2005) 10908-10918.

[8] E Laredo: $6^{\text {th }}$ Int. Conf. on Broadband Dielectric Spectroscopy and its Applications, Book of Abstracts, Madrid, Spain (2010) 121.

[9] D. Wu, Y. Yang, M. Zhang, W. Yu: Biomolecules, 10, (2009) 417; A. Bello, M. Grimao, E. Laredo, D. Wu: $6^{\text {th }}$ Int. Conf. on Broadband Dielectric Spectroscopy and its Applications, Book of Abstracts, Madrid, Spain (2010) 287.

[10] V.V. Meriakri, I.A. Chmutin, N.V. Rivkina, S. Zhou, Materials of Int. Conf. ACN'2911 Advanced Carbon Nanomaterials, P 2.29, S. (2011) St. Petersburg, Russia 137.

[11] Arrese-Igor, A, Alegrio, J. Moreno, J. Colmenaro: Macromolecules (2010) 44, 3611.

[12] W. Coffey, Yu. Kalmykov, S. Titov: NATO Advanced
Research Workshop "Broadband Dielectric Spectroscopy and its Advanced Technological Application”, Perpignan, France, (2011), 22.

[13] K. Fukshima, C. Abbate, D. Iobuani, M. Gennari, G. Camjno: Polymer Degrad. Stabil. (2009) 94, 1646-1655.

[14] K. Fukshima, D. Iobuani, C. Abbate, M. Areno, P. Pizzerelli: Polymer J. (2011) 47, 139-152.

[15] C. Zhang, H. Lu: J. Material Science, Materials in Medicine (2010) 21, 3077-3081.

[16] O. Russo, B. Vetrano, D. Aciemo $6^{\text {th }}$ Int. Conf. on Times of Polymers (TOP) and Composites AIP Conference Proceedings 1459 (2012), Ischia, Italy, 23-25.

[17] D. Kalenov, V. Meriakri, M. Parkhomenko Sh. Zhou: $6^{\text {th }}$ Int. Conf. on Times of Polymers (TOP) and Composites AIP Conference Proceedings 1459 (2012), Ischia, Italy, 77-79.

[18] Bourbigot, M. Delichatios, V. Meriakri, I .Nikitin, M. Parkhomenko: Conference STEPI 8,(2008) Montpepier, France P 47.

[19] B. Bisceglia, P. Leo, A. Pastore, S. von Gratowski, V. Meriakri: Journal of Microwave Power and Electromagnetic Energy (2011) 45 (1) 1-13.

[20] V.V. Meriakri, E.E. Chigrai, M.P. Parkhomenko: $6^{\text {th }}$ Int. Conf. on Broadband Dielectric Spectroscopy and Its Applications, Book of Abstracts, Madrid, Spain (2010) 163.

[21] V.V. Meriakri, M.P. Parkhomenko, E.E. Chigryai, I.P. Nikitin, C.L. Pan, R.P. Pan: 2010 Int. Kharkov Symposium on Physics and Engineering of Microwaves, Millimeter and Submillimeter Waves, IEEE Catalog Number: CFP10780-CDR, ISBN: 978-1-4244-7898-9, INV-4, Kharkov, Ukraine (2010) 10-14.

[22] V. Meriakri, E. Chigryai, and I.P. Nikitin: Int. $\mathrm{THz}$ Conference, Villach, Austria (2011) 143-147.

[23] V.V. Meriakri, E.E. Chigrai, I.P. Nikitin, M.P. Parkhomenko, S.V. von Gratowskl: NATO Advanced Research Workshop "Broadband Dielectric Spectroscopy and Its Advanced Technological Application," Perpignan, France ( 2011) 48.

[24] V. Meriakri, E. Chigryai, M. Parkhomenko, R. Denisyuk, S. von Gratowski: Journal of Biofuels (2011) 2 (1), 8-13.

[25] V.V. Meriakri, M.P. Parkhomenko, E.E. Chigryai, I.P. Nikitin, C.L. Pan, R.P. Pan, Liquid Crystals (2010) 37 (11), 1453-1457.

[26] Xiaotong Zheng, Shaobing Zhou, Yu Xiao, Xiongjun Yu, Xiaohong Li, PeizhuoWu, Colloids and Surfaces B: Biointerfaces (2009) 71, 67-72.

[27] J.L.E. Ivirico, M.S. Sánchez, R. Sabater i Serra, J.M.M. Dueñas, J.L.G. Ribelles, M.M. Pradas: Macromol. Chem. Phys. (2006) 207, 2195-2205.

[28] S. Bourbigot, G. Fontaine, A. Gallos, S. Bellayer, Polymers for Advanced Technologies (2011) 22 (1), 30-37.

[29] O.V. Betskii, N.D. Devyatkov and V.V. Kislov: Biomedical Radio Engineering (1998) 4, 19-29 (in Russian). 